- Original Article

\title{
Association between the Eating Family Meal and the Prevalence of Metabolic Syndrome Using Data from Korea National Health and Nutrition Examination Survey (2007-2012)
}

\author{
Shin-Ae Park, Woo-Chul Park, Yu-Jin Kwon, Jae-Yong Shim* \\ Department of Family Medicine, Yonsei University College of Medicine, Seoul, Korea
}

Background: Several studies have shown that family meals promote a well-balanced and healthier diet and weight status. Metabolic syndrome is related to eating behavior. This study investigated the association between eating family meals and the prevalence of metabolic syndrome.

Methods: This cross-sectional study included 4,529 subjects who participated in the Korea National Health and Nutrition Examination Survey IV and V (2007-2012). A self-reported questionnaire was used to assess dietary status. Metabolic syndrome was defined according to the guidelines of the modified version of the National Cholesterol Education Program Adult Treatment Panel III. We compared the overall quality of dietary intake in family meal. Results: Nutritional adequacy ratios for energy, protein, calcium, vitamin $A$, vitamin $B_{1}$, vitamin $B_{2}$, vitamin $C$, niacin, and potassium, and the mean adequacy ratio were significantly higher in the family meal group $(\mathrm{P}<0.05)$. The prevalence of metabolic syndrome was lower in the family meal group $(\mathrm{P}<0.05)$. However, we observed no significant association between eating family meals and the prevalence of metabolic syndrome.

Conclusion: This study demonstrated that eating family meals appeared to be associated with nutrient adequacy. However, we observed no significant differences in prevalence of metabolic syndrome between the 2 groups.

Keywords: Meal; Nutritional Status; Social Class; Metabolic Syndrome 


\section{INTRODUCTION}

In the last 60 years, Korea's rapid modernization and industrialization have westernized the country. Rapid industrialization brought about the breakdown of the family system, and led to an increase in the number of nuclear families and single households. ${ }^{1)}$ According to Statistics Korea, single households constituted $9.1 \%$ of the total in 1990, $12.7 \%$ in $1995,15.5 \%$ in $2000,19.9 \%$ in 2005 , and $23.8 \%$ in 2010 . The number is predicted to reach $31.3 \%$ by $2025 .{ }^{2)}$

Family meals refer to meals eaten together with family members. The percentage of those eating family meals is decreasing annually, along with the increase in single households. Statistics Korea reported that the percentage of those eating family meals of breakfast, lunch, or dinner was $56.3 \%, 21.1 \%$, and $68.3 \%$, respectively, in 2007 . The percentages fell to $46.1 \%, 14.4 \%$, and $65.1 \%$, respectively, in $2013 .{ }^{3)}$

Compared to eating alone, eating a family meal is associated with healthy dietary intake, including more fruits and vegetables, and less fried foods and carbonated beverages. Eating a family meal is also associated with higher intake of nutrients, including fiber, calcium, folate, and iron, and vitamins $\mathrm{B}_{6}, \mathrm{~B}_{12}, \mathrm{C}$, and E. ${ }^{4,5)}$ Preparing meals for oneself may lead to inadequate dietary variety because of the use of fewer food ingredients and a lack of motivation to prepare high quality meals every day. ${ }^{6)}$ In fact, divorced or bereaved spouses ate less vegetables compared with those who were married. ${ }^{7,8}$ Elderly adults who eat alone are at higher risk of nutritional deficiency, poor weight status, ${ }^{9)}$ and death. ${ }^{10)}$ Some studies reported that decreased frequency of eating family meals is more likely to cause overweight. ${ }^{11)}$ Eating family meals was shown to be a protective factor for weight control in adolescence. $^{12)}$

The prevalence of metabolic syndrome has increased significantly in Korea in the past 10 years. ${ }^{13)}$ Metabolic syndrome is related to lifestyle factors such as drinking, smoking, exercise, and eating behaviors. ${ }^{14,15)}$ Several studies on the relationship between eating behavior and metabolic syndrome reported that higher intake of carbohydrate and lower intake of fiber were associated with an increase in its prevalence. $^{16)}$

The first aim of this study is to compare the quality of dietary intake in a family meal group and a non-family meal group. Based on the hy- pothesis that metabolic syndrome may be related to nutrient intake, this study also investigated the association between eating a family meal and the prevalence of metabolic syndrome, using data of the Korea National Health and Nutrition Examination Survey (KNHANES) IV and V, conducted from 2007 to 2012.

\section{METHODS}

\section{Study Sample}

This study was based on data from the KNHANES IV and V (20072012). The KNHANES is a cross-sectional, nationwide survey conducted by the Korean Ministry of Health and Welfare. The KNHANES is composed of 3 sections: a health interview, health examination, and a nutrition survey. In the KNHANES IV (2007-2009) and V (20102012), 50,405 individuals participated in the examination. This study excluded participants younger than 19 years of age or older than 64 years of age. Those who skipped at least 1 meal out of 6 during the 2 days prior to the health examination, and those who went out to eat more than 3 or 4 times a week were excluded from this study. We also excluded subjects with missing data for laboratory tests, diet status, and nutrition intake, those who did not fast more than 8 hours before blood sampling, and those with a diagnosis of any malignancy. Finally, 4,529 subjects were included in the statistical analysis. All participants in the survey provided informed written consent (Figure 1).

\section{General Characteristics of the Subjects}

Physical examinations were performed by trained experts following standardized procedures. The height and body weight were measured to the nearest $0.1 \mathrm{~cm}$ and $0.1 \mathrm{~kg}$, respectively. Body mass index (BMI) was calculated as weight $(\mathrm{kg}) /$ height squared $\left(\mathrm{m}^{2}\right)$. Waist circumference was measured at the narrowest location between the iliac crest and the lower border of the rib cage. Systolic blood pressure and diastolic blood pressure were measured on the right arm using a standard mercury sphygmomanometer (Baumanometer; WA Baum Co. Inc., Copiague, NY, USA). Two systolic and diastolic blood pressure measurements were collected at 5-minute intervals and averaged for analysis. To assess serum levels of biochemical markers, blood samples were collected from antecubital veins after an overnight fast. Monthly
45,876 were excluded.

21,214 were $<19$ years or $\geq 65$ years of age.

3,854 did not answer three question about their family meal practice

7 skipped at least one meal out of the last six meals prior to the survey.

17,449 ate out more than three or four times a week

580 did not fast more than 8 hour before blood sampling.

103 diagnosed any malignancy.

2,669 with missing data for anthropometric measurements, biochemical measurements or nutrient intake.
Figure 1. Subject eligibility. KNHANES, Korea National Health and Nutrition Examination Survey. 
income was divided into quartiles as follows: lower, lower middle, upper middle, or highest. Education level was divided into 4 groups as elementary school, middle school, high school, or university, by the subject's highest achieved level. Marital status was divided into 2 categories of single or married.

Regular drinkers were defined as individuals who drank alcohol at least once a month. Subjects who smoked cigarettes during the survey period were defined as current smokers. Regular exercise was defined as $\geq 20$ minutes of vigorous-intensity physical activity $\geq 3$ days a week or $\geq 30$ minutes of moderate-intensity physical activity $\geq 5$ days a week. ${ }^{17)}$

\section{Dietary Assessment}

Nutrient intake data were obtained from the nutrition survey in the KNHANES (IV-V). The nutrition questionnaire was based on the 24hour recall method. To estimate the status of nutrient intake, the nutrient adequacy ratio (NAR) was calculated as the ratio of the age/gender-specific recommended nutrient intake or adequate intake. NAR was truncated at 1 if the value was over 1 . The mean adequacy ratio (MAR) was obtained from the average NAR for 10 selected nutrients (i.e., protein, calcium, iron, fiber, niacin, and potassium, and vitamins $\mathrm{A}, \mathrm{B}_{1}, \mathrm{~B}_{2}$, and $\mathrm{C} .{ }^{18)}$

\section{Definition of Family Meal}

The KNHANES contained the following 3 questions, to be answered yes or no: "Have you eaten breakfast with your family in the past year?", "Have you eaten lunch with your family in the past year?", and "Have you eaten dinner with your family in the past year?" The non-family meal group consisted of subjects who answered no to all 3 questions. Subjects who answered yes to any of the questions were included in the family meal group.

In order to prevent any error due to the combined absence of family meals and skipping of meals, those who skipped at least 1 out of the 6 meals prior to the nutrition survey were excluded. In order to avoid the confounding variable of eating out, subjects included those only who ate out no more than twice per week.

\section{Definition of Metabolic Syndrome}

Metabolic syndrome was defined according to the guidelines of the modified version of the National Cholesterol Education Program Adult Treatment Panel III. ${ }^{19)}$ Metabolic syndrome was defined as the presence of 3 or more of the following criteria: (1) waist circumference $\geq 102 \mathrm{~cm}$ for men and $\geq 88 \mathrm{~cm}$ for women; (2) systolic blood pressure $\geq 130 \mathrm{~mm} \mathrm{Hg}$ or diastolic blood pressure $\geq 85 \mathrm{~mm}$ Hg or taking any antihypertensive medication; (3) fasting plasma glucose levels $\geq 100 \mathrm{mg}$ / $\mathrm{dL}$ or taking any antidiabetic medication; (4) triglyceride levels $\geq 150$

Table 1. Subject characteristics according to eating with family

\begin{tabular}{|c|c|c|c|}
\hline Characteristic & Non-family meal group & Family meal group & P-value \\
\hline Sex (men) & $502(28.5)$ & $4,027(24.5)$ & 0.052 \\
\hline Age (y) & $52.3 \pm 10.1$ & $49.0 \pm 10.9$ & $<0.001$ \\
\hline Body mass index $\left(\mathrm{kg} / \mathrm{m}^{2}\right)$ & $23.8 \pm 3.4$ & $23.7 \pm 3.3$ & 0.354 \\
\hline Waist circumference (cm) & $81.4 \pm 10.0$ & $80.4 \pm 9.6$ & 0.046 \\
\hline Systolic blood pressure (mm Hg) & $120.3 \pm 18.0$ & $118.4 \pm 17.2$ & 0.016 \\
\hline Diastolic blood pressure (mm Hg) & $77.4 \pm 10.6$ & $76.1 \pm 10.3$ & 0.009 \\
\hline Fasting plasma glucose (mg/dL) & $98.9 \pm 20.9$ & $97.3 \pm 22.9$ & 0.132 \\
\hline Triglyceride (mg/dL) & $132.2 \pm 90.2$ & $126.2 \pm 95.5$ & 0.179 \\
\hline High-density lipoprotein cholesterol (mg/dL) & $53.1 \pm 12.8$ & $52.7 \pm 12.9$ & 0.583 \\
\hline Total cholesterol & $194.9 \pm 38.7$ & $190.0 \pm 37.2$ & 0.005 \\
\hline White blood cell (cells/mL) & $5.8 \pm 1.6$ & $5.8 \pm 1.7$ & 0.904 \\
\hline Calories $(\mathrm{kcal} / \mathrm{d})$ & $1,939.5 \pm 852.0$ & $1,936.3 \pm 731.8$ & 0.927 \\
\hline Household income & & & 0.029 \\
\hline Lowest & 35.7 & 29.3 & \\
\hline Lower middle & 24.3 & 26.8 & \\
\hline Upper middle & 23.1 & 24.2 & \\
\hline Highest & 16.9 & 19.7 & \\
\hline Education level (y) & & & $<0.001$ \\
\hline$<7$ & 35.3 & 26.6 & \\
\hline $7-9$ & 15.5 & 14.7 & \\
\hline $10-12$ & 33.7 & 34.0 & \\
\hline$\geq 13$ & 15.5 & 24.7 & \\
\hline Regular drinker & 46.0 & 43.0 & 0.196 \\
\hline Current smoker & 20.5 & 13.6 & $<0.001$ \\
\hline Regular exercise & 49.8 & 49.9 & 0.954 \\
\hline Marital status & 90.0 & 95.7 & $<0.001$ \\
\hline Metabolic syndrome & 27.5 & 20.6 & $<0.001$ \\
\hline
\end{tabular}

Values are presented as number (\%), mean \pm standard error, or \%. Differences were determined using Student t-test for continuous variables and the chi-square test for categorical variables $(P<0.05)$. 
$\mathrm{mg} / \mathrm{dL}$; and (5) high-density lipoprotein cholesterol levels $\leq 40 \mathrm{mg} / \mathrm{dL}$ for men and $\leq 50 \mathrm{mg} / \mathrm{dL}$ for women.

\section{Statistical Analysis}

Statistical analyses were performed using IBM SPSS ver. 21.0 (IBM Corp., Armonk, NY, USA). Continuous variables were expressed as mean \pm standard error and analyzed using Student t-test. Categorical variables were described as percentage of subjects and were analyzed using chi-square tests. The odds ratio (OR) and 95\% confidence interval (CI) for metabolic syndrome were calculated using multiple logistic regression analysis after adjusting for sex, age, income, education, occupation, marital status, drinking, smoking, exercise, daily caloric intake, white blood cell count, and BMI. For all analyses, P-values $<0.05$ were considered statistically significant.

\section{RESULTS}

The average waist circumference was smaller in the family meal group (Table 1). The subjects in the family meal group showed significantly lower levels of blood pressure and total cholesterol and had a higher percentage with high income compared with the non-family meal group. In addition, individuals in the family meal group were more likely to exercise and had a lower percentage of current smokers. The percentage of those who were married was higher in the family meal group. The prevalence of metabolic syndrome was lower in the family meal group (all P-values <0.05) (Table 1).

To evaluate meal quality, we compared the NAR and MAR in the family meal group and the non-family meal group (Table 2 ). The family meal group had more intake of energy, protein, calcium, vitamin $\mathrm{A}$, vitamin $B_{1}$, vitamin $B_{2}$, vitamin $C$, niacin, and potassium, and a higher $\operatorname{MAR}(\mathrm{P}<0.05)$.

In multiple logistic analysis, the OR $(95 \% \mathrm{CI})$ for the family meal group was 0.68 (0.55-0.84) without adjusting for other variables. After adjusting for sex and age, the OR (95\% CI) for metabolic syndrome was not significant in model 2 (0.82 [0.66-1.02]) (Table 3).

Table 2. The nutrient adequacy ratio and mean adequacy ratio

\begin{tabular}{lccr}
\hline \multicolumn{1}{c}{ Variable } & Non-family meal group & Family meal group & P-value \\
\hline Energy & $0.85 \pm 0.18$ & $0.86 \pm 0.16$ & 0.034 \\
Protein & $0.93 \pm 0.14$ & $0.95 \pm 0.12$ & $<0.001$ \\
Calcium & $0.64 \pm 0.28$ & $0.67 \pm 0.26$ & 0.028 \\
Iron & $0.90 \pm 0.19$ & $0.89 \pm 0.18$ & 0.655 \\
Fiber & $0.37 \pm 0.22$ & $0.38 \pm 0.21$ & 0.134 \\
Vitamin A & $0.73 \pm 0.31$ & $0.78 \pm 0.28$ & $<0.001$ \\
Vitamin $B_{1}$ & $0.83 \pm 0.20$ & $0.87 \pm 0.18$ & $<0.001$ \\
Vitamin $B_{2}$ & $0.73 \pm 0.26$ & $0.77 \pm 0.24$ & $<0.001$ \\
Vitamin C & $0.73 \pm 0.30$ & $0.78 \pm 0.27$ & $<0.001$ \\
Niacin & $0.83 \pm 0.20$ & $0.86 \pm 0.18$ & $<0.001$ \\
Potassium & $0.75 \pm 0.24$ & $0.78 \pm 0.22$ & 0.005 \\
Mean adequacy ratio & $0.74 \pm 0.18$ & $0.77 \pm 0.16$ & $<0.001$ \\
\hline
\end{tabular}

Values are presented as mean \pm standard error. Significance determined by Student t-test $(P<0.05)$.

\section{DISCUSSION}

This study investigated the association between eating a family meal and nutritional adequacy, and between eating a family meal and the prevalence of metabolic syndrome.

The family meal group showed significantly lower measurements of waist circumference, systolic blood pressure, diastolic blood pressure, and cholesterol level. NAR was calculated to determine the nutrient adequacy. The family meal group showed significantly higher intake of energy, protein, calcium, niacin, and potassium, and vitamins $A, B_{1}$, $\mathrm{B}_{12}$, and $\mathrm{C}$. The MAR was also higher in this group. Therefore, eating family meals meant eating more balanced meals.

A prior study showed that higher intake of carbohydrate was associated with an increased prevalence of metabolic syndrome. ${ }^{16)}$ In this study, the level of energy intake was higher in the family meal group compared to the non-family meal group. However, the OR (95\% CI) for metabolic syndrome was $0.68(0.55-0.84)$ in the family meal group without any adjustment, and was 0.81 (0.64-1.03) with adjustment for age, sex, income, education, marital status, drinking, smoking, exercise, daily energy intake, white blood cell count, and BMI. Therefore, in spite of higher energy intake in the family meal group, there was no statistically significant association between eating family meals and the prevalence of metabolic syndrome.

Several studies reported that higher levels of several antioxidants in serum are conversely associated with metabolic syndrome risk. ${ }^{20,21)} \mathrm{In}$ dividuals in the family meal group had more antioxidant vitamins compared to those in the non-family meal group. Even though eating family meals resulted in more energy intake, there were no significant differences in prevalence of the metabolic syndrome between the groups. We assumed that this may be related to the higher intake of antioxidant vitamins in the family meal group.

Our study has some limitations. First, since this study is based on cross-sectional data obtained from the KNHANES IV and V, the relationship between eating family meals and metabolic syndrome remains unclear. Second, it is highly likely that the survey participants could have given recall-biased information due to time differences between information retrieval and the actual experience. Third, the question about having family meals was formulated to find out if a participant had breakfast, lunch, and dinner during the prior 1 year. However, the question about skipping meals was formulated to find

Table 3. Odds ratios for metabolic syndrome according to eating with family

\begin{tabular}{lcc}
\hline & Non-family meal group & Family meal group \\
\hline Not adjusted & 1 & $0.68(0.55-0.84)$ \\
Model 1 & 1 & $0.82(0.66-1.02)$ \\
Model 2 & 1 & $0.84(0.68-1.05)$ \\
Model 3 & 1 & $0.81(0.64-1.03)$ \\
\hline
\end{tabular}

Values are presented as odds ratio (95\% confidence interval). Model 1: adjusted for sex and age; model 2: adjusted for sex, age, income, education, and marital status; model 3: adjusted for sex, age, income, education, marital status, drinking, smoking, exercise, daily caloric intake, white blood cell, and body mass index. 
out if a participant had breakfast, lunch, and dinner for only the 2 days prior to the nutrition survey. For this reason, although only those who had all 6 meals during the 2 days prior to the nutrition survey were selected as appropriate subjects, there is insufficient evidence that subjects usually had 3 meals per day. Lastly, this study showed that subjects in the non-family meal group had many health-related risk factors that were statistically significant. Although these risk factors were adjusted in multiple logistic analysis, there is a possibility that risk factors that were not covered in this study were present.

In conclusion, this study showed that eating family meals appeared to be associated with more balanced dietary intake, and that energy intake in the family meal group was higher than in the non-family meal group, but the prevalence of metabolic syndrome was not significantly higher than in the non-family meal group.

\section{CONFLICT OF INTEREST}

No potential conflict of interest relevant to this article was reported.

\section{REFERENCES}

1. Bae HO. A study on one person households in Korea. J Popl Assoc Korea 1993;16:125-39.

2. Statistics Korea. Complete enumeration results of the 2010 population and housing census. Daejeon: Statistics Korea; 2011.

3. Statistics Korea. Ministry of Health, Welfare and Family. Daejeon: Statistics Korea; 2015.

4. Gillman MW, Rifas-Shiman SL, Frazier AL, Rockett HR, Camargo CA Jr, Field AE, et al. Family dinner and diet quality among older children and adolescents. Arch Fam Med 2000;9:235-40.

5. Fink SK, Racine EF, Mueffelmann RE, Dean MN, Herman-Smith R. Family meals and diet quality among children and adolescents in North Carolina. J Nutr Educ Behav 2014;46:418-22.

6. Tani Y, Sasaki Y, Haseda M, Kondo K, Kondo N. Eating alone and depression in older men and women by cohabitation status: the JAGES longitudinal survey. Age Ageing 2015;44:1019-26.

7. Lee S, Cho E, Grodstein F, Kawachi I, Hu FB, Colditz GA. Effects of marital transitions on changes in dietary and other health behaviours in US women. Int J Epidemiol 2005;34:69-78.

8. Eng PM, Kawachi I, Fitzmaurice G, Rimm EB. Effects of marital transitions on changes in dietary and other health behaviours in US male health professionals. J Epidemiol Community Health 2005;59:56-62.

9. Tani Y, Kondo N, Takagi D, Saito M, Hikichi H, Ojima T, et al. Com- bined effects of eating alone and living alone on unhealthy dietary behaviors, obesity and underweight in older Japanese adults: results of the JAGES. Appetite 2015;95:1-8.

10. Sahyoun NR, Jacques PF, Dallal GE, Russell RM. Nutrition Screening Initiative Checklist may be a better awareness/educational tool than a screening one. J Am Diet Assoc 1997;97:760-4.

11. Gable S, Chang Y, Krull JL. Television watching and frequency of family meals are predictive of overweight onset and persistence in a national sample of school-aged children. J Am Diet Assoc 2007;107:5361.

12. Berge JM, Wall M, Hsueh TF, Fulkerson JA, Larson N, Neumark-Sztainer D. The protective role of family meals for youth obesity: 10-year longitudinal associations. J Pediatr 2015;166:296-301.

13. Lim S, Shin H, Song JH, Kwak SH, Kang SM, Yoon JW, et al. Increasing prevalence of metabolic syndrome in Korea: the Korean National Health and Nutrition Examination Survey for 1998-2007. Diabetes Care 2011;34:1323-8.

14. Park HS, Oh SW, Cho SI, Choi WH, Kim YS. The metabolic syndrome and associated lifestyle factors among South Korean adults. Int J Epidemiol 2004;33:328-36.

15. Woolf SH, Jonas S, Kaplan-Liss E. Health promotion and disease prevention in clinical practice. 2nd ed. Philadelphia (PA): Lippincott Williams \& Wilkins; 2008.

16. Carnethon MR, Loria CM, Hill JO, Sidney S, Savage PJ, Liu K, et al. Risk factors for the metabolic syndrome: the Coronary Artery Risk Development in Young Adults (CARDIA) study, 1985-2001. Diabetes Care 2004;27:2707-15.

17. Cho Y, Shin MJ, Chung HK. Effects of diet modification on meal quality and quality of life in korean diabetic patients: data from Korea national health and nutrition examination survey (2007-2011). Clin Nutr Res 2014;3:106-14.

18. The Korean Nutrition Society. Dietary reference intakes for Koreans 2010. Seoul: The Korean Nutrition Society; 2010.

19. Grundy SM, Cleeman JI, Daniels SR, Donato KA, Eckel RH, Franklin BA, et al. Diagnosis andmanagement of the metabolic syndrome: an American Heart Association/National Heart, Lung, and Blood Institute scientific statement. Circulation 2005;112:2735-52.

20. Beydoun MA, Shroff MR, Chen X, Beydoun HA, Wang Y, Zonderman $A B$. Serum antioxidant status is associated with metabolic syndrome among U.S. adults in recent national surveys. J Nutr 2011;141:903-13.

21. Czernichow S, Vergnaud AC, Galan P, Arnaud J, Favier A, Faure H, et al. Effects of long-term antioxidant supplementation and association of serum antioxidant concentrations with risk of metabolic syndrome in adults. Am J Clin Nutr 2009;90:329-35. 